Скопје, Македонија

\title{
ONE IMPLEMENTATION OF MATHEMATICAL MORPHOLOGY IN MEDICAL (ECG) APPLICATION
}

\author{
ROMAN GOLUBOVSKI, GJORGJI MARKOSKI, ELENA GOLUBOVSKA, \\ AND VASKO KOKALANOV
}

\begin{abstract}
Acquired ECG signal is degraded by low-pass EMG drift modifying its baseline; and by power net interference, high-pass noise and $\mathrm{A} / \mathrm{D}$ conversion compromising its morphology. One of the main problems in the automated ECG analysis is to filter out the baseline wander, and extract the isoelectric reference, thus enabling accurate measurements and morphology recognition. Using Mathematical Morphology (MM) binary primitive operators, accurate baseline extraction is performed in three steps. First is to determine the exact heart rate (HR), which is done by high-pass MM filtering and R-wave detection. In the second step, preliminary baseline estimation is performed using low-pass MM filtering, thus allowing accurate morphology recognition. In the third step, the baseline is corrected using the ending points of TP intervals (P-start) which are considered as truly isoelectric. This procedure allows accurate baseline extraction and recognition of the complete morphology. The Mathematical Morphology offers a reliable solution for the baseline extraction problem, allowing ECG analysis for holter and monitoring applications.
\end{abstract}

\section{Introduction to Mathematical Morphology (MM)}

Mathematical morphology is a discipline of image analysis, introduced in the 1960s by Georges Matheron and Jean Serra. Historically, this was the first consistent nonlinear image analysis theory, which included not only theoretical results but also many practical aspects.

In this paper the work is done in real plane $\mathbb{R}^{2}$ with usual operations and relations. So all definitions are given for $\mathbb{R}^{2}$. More details regarding MM, its applications and algorithms are given in [1], [2] and [3]. This implementation works with binary images. i.e. image (subset of $\mathbb{R}^{2}$ ) that possess only two levels: strictly black and strictly white.

Structuring elements are families of special sets $B$ that are known a priori and can be adapted to specific needs (in terms of size, orientation, etc.). They allow definition of operators in a practical way.

2000 Mathematics Subject Classification. 92C50, 92C20, 91E10.

Key words and phrases. Mathematical Morphology, opening, closing, ECG signals. 
Let $X$ be a binary image and $p \in \mathbb{R}^{2}$. Structuring elements are also binary images. One point of $B$ can be fixed and called origin of $B$.

Translation of $X$ by $p$ is the set $X_{p}=\{x+p \mid x \in X\}$. Here $p$ is a translation vector.

Dilatation of $X$ by $B$ is defined by $\delta_{B}(X)=X \oplus B=\bigcup_{b \in B} X_{b}$.

Obviously that holds $X \oplus B=\bigcup_{x \in X} B_{x}=\{x+b \mid x \in X, b \in B\}$.

Erosion of $X$ by $B$ is the set $\varepsilon_{B}(X)=X \ominus B=\bigcap_{b \in B} X_{-b}=\left\{p \in \mathbb{R}^{2} \mid B_{p} \subseteq X\right\}$.

Opening of $\mathrm{X}$ by structuring element $\mathrm{B}$ is $\gamma_{B}(X)=X \circ B=(X \ominus B) \oplus B$ and

Closing of $\mathrm{X}$ by $\mathrm{B}$ is defined by

$$
\varphi_{B}(X)=X \bullet B=(X \oplus B) \ominus B .
$$

Choosen structuring element is the flat segment $\{(x, 0) \mid-a \leq x \leq a\}$, where $a \in \mathbb{R}, a>0$.

\section{The Implementation Domain}

The need for electrocardiographic (ECG) diagnosis automation and the development of appropriate expert systems is as old as the introduction of electric apparatus in cardiology. The development process had been accelerated with the introduction of microcomputers which overcame the limits of the delicate, often inferior and sometimes impossible hardware application. This apparent rapid development is supported by an upsurge of creativity in the field of ECG processing. The problems that modern diagnostic expert systems face are in the domain of R-wave identification. With adequate $\mathrm{R}$ wave detection we are then able to determine almost all of the components of the heart cycle, such as heart rate (HR), RR (interval) and heart rate variability (HRV). Furthermore we are then able to perform various statistic analysis; elimination of complex net and environmental interference, as well as the EMG artifacts; baseline wander removal; accurate morphology identification and relevant parameters measurement. Today, specialized surveillance algorithms in implantable devices (pacemakers, defibrillators) allow improvement in the quality of normal life in people depended on these devices during $24 \mathrm{~h}$, while safely away from safe Coronary Care Units (CCU). In spite of these improvements, modern science and technology have not been able to successfully demonstrate an integral solution embracing the complete ECG diagnostics, thus producing specialized diagnostic devices for different aspects of the ECG analysis based on specific requirements. Attempts have been made to utilize MM in heartbeat classification [4] and adaptive fiducial point extraction [5]. MM approach had been compared as efficient methodology of noise reduction [6], and proposed as a baseline wander filter [7]. This paper presents a Mathematical 
Original Signal

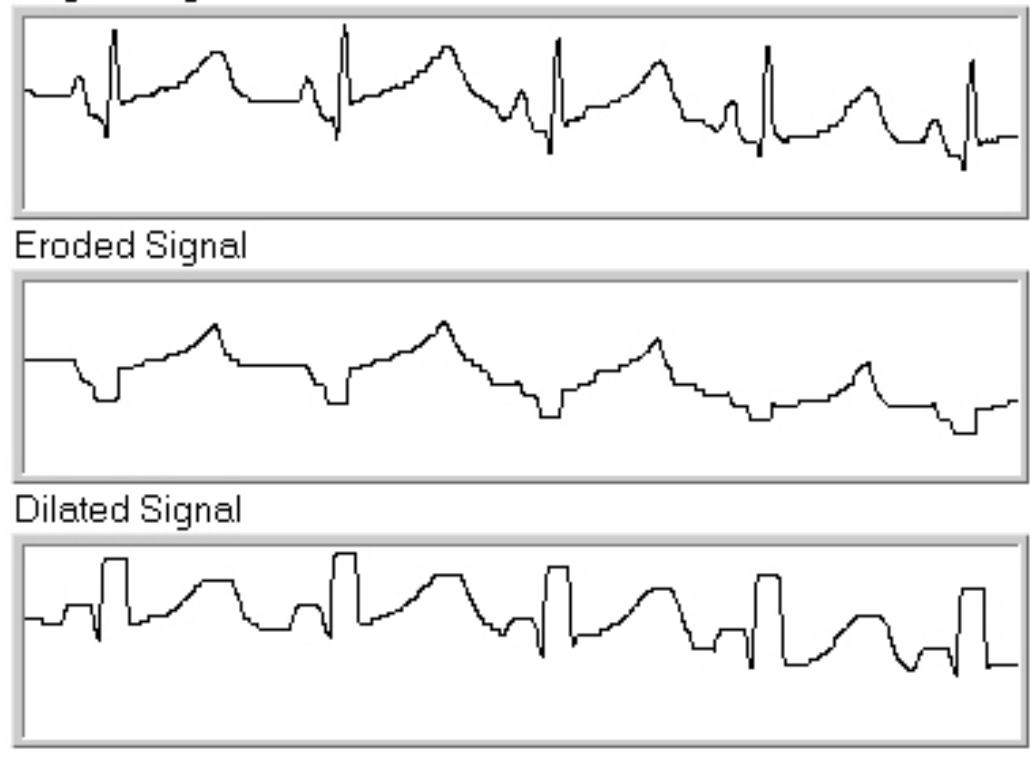

Figure 1: Erosion and dilation of ECG signal

Morphology (MM) method for accurate Digital Signal Processing (DSP) of ECG signals and their analysis.

\section{The MM Application}

The Morphology Filter (MF) for ECG strip analysis is intended for a 24h surveillance of the ST segment elevation as well as the rest of the ECG parameters in $\mathrm{CCU}$ patients. For testing purpose, the acquired signals were not filtered against net interference $(50 / 60 \mathrm{~Hz})$, EMG Low-Pass drift and baseline wander. Other characteristics are: extremely high HR (babies) and low Analog-to-Digital Conversion (ADC) quality. The MF is intended for 1D signals, which for the purpose of MM aplication are transformed into 2D images by involving the umbra below the ECG signal, where the signal itself is the upper border of the image object of interest. Binary morphology operators are used. The desired characteristic for extraction from the original signal is its baseline wander (isoelectric reference), so a flat structuring element (SE) is chosen. Figure 1 illustrates the shrinking and expanding effects of the applied erosion and dilation on the original signal respectively (SE is not optimized).

Figure 2 illustrates the effects of both when applied on the same original. Obviously, the opening of the signal implies sliding of the SE along it and 


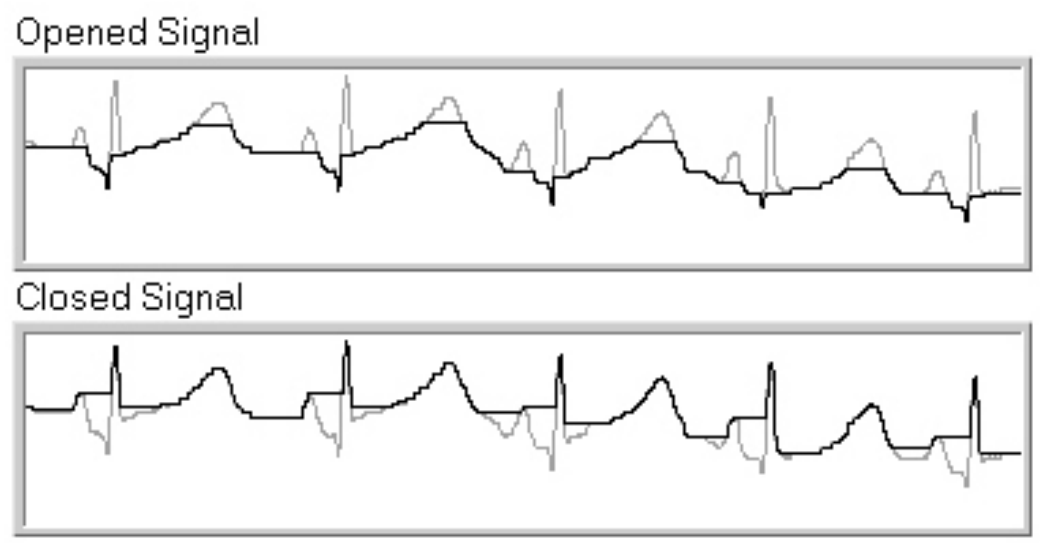

Figure 2: Opening and closing of ECG signal

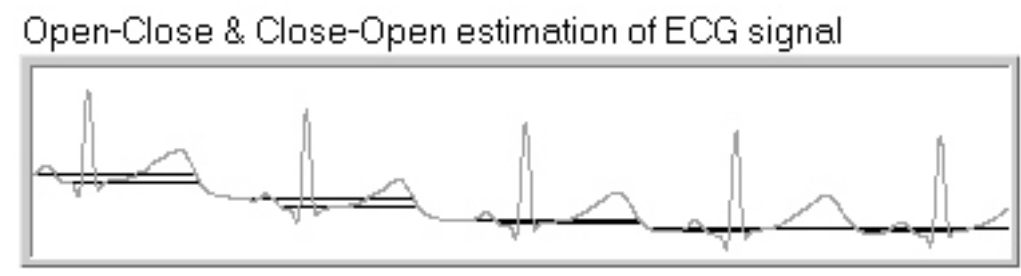

Figure 3: Open/Close baseline estimationl

cutting off its peaks; and the closing of the signal implies filling up its valleys. The results depend on the size of the SE, thus consecutive application of opening and closing in either order will result in attenuation of parts of the spectrum just like standard Low/High/Band-Stop/Pass filter.

Figure 3 illustrates both the opening-closing and closing-opening combinations. Clearly, both oscillate around the desired baseline and obviously their average is a better estimation of the isoelectric reference but still far from perfect. Eventhough it is not perfect, this preliminary estimation of the baseline allows absolutely reliable R-wave thresholding, and thus HR determination. Having the exact HR, the optimal length for the SE can be calculated and a better (secondary) baseline estimation can be performed for final correction using corrective points on the ECG signal.

Figure 4 shows a block diagram of the solution.

Detailed algorithm for MM method for ECG processing is as follows:

- assume initial $\mathrm{HR}=60 \mathrm{bpm}$ and calculate SE length

- extract QRS signal (High-Pass MM filtering)

- mark Rs and calculate actual HR by thresholding 


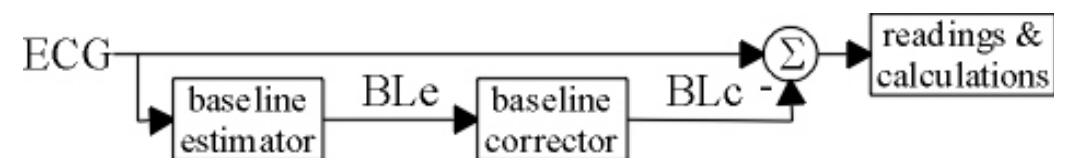

Figure 4: Morphology filter block diagram

- calculate optimal SE according actual HR

- if $\mathrm{S}$ is dominant in the scanned lead then threshold the $\mathrm{S}$ deflections, check the HR and mark all the $\mathrm{R}$ waves (left from corresponding $\mathrm{S}$ deflections)

- extract preliminary baseline-BLe (Low-Pass filter)

- form new signal: PQRST=ECG-BLe

- determine all P, Q, R, S and T amplitudes' positions as well as Pstart, Pend and Tend positions using derivative routines

- taking the TP segment as truly isoelectric, correct all Pstart positions as well as the baseline (BLc)

- correct all Pend and Tend positions accordingly

- determine all J-point positions using a derivative routine and then the corresponding ST segment measurement positions

- read marked amplitudes

- calculate required intervals.

Figure 5 illustrates the High-Pass MM filtering of the ECG signal for the purpose of QRS signal thresholding and HR calculation. The SE size (length) is now chosen to correspond to the width of the objects of the P-QRS-T cycle corresponding to the higher frequencies of the signal's spectrum - the P and QRS waves. Having in mind their normal duration (not exceeding $120 \mathrm{~ms}$ ) and normal heart rates of $60-120 \mathrm{bpm}$, the SE is sized $9 \%$ of cycle's duration (experimentally determined optimum). If the lead has dominant $\mathrm{S}$ deflections and $\mathrm{R}$ waves inferior comparing to both the $\mathrm{P}$-waves and the T-waves. Standard thresholding would probably double the HR due to the $\mathrm{P}$ and $\mathrm{T}$ domination over $\mathrm{R}$. In such signals the absolute value of the minimal value $(\max \mathrm{S}$ ) in the ECG strip is always bigger than $50 \%$ of the maximal value $(\max R$ ) which is a positive identification for "dominant $\mathrm{S}$ over R" type of lead. In such cases HR is determined by thresholding the $\mathrm{S}$ deflections and R-wave positions are determined left from corresponding S deflections.

Figure 6 illustrates the Low-Pass MM filtering of the ECG signal producing the preliminary baseline estimation (BLe). The SE is now chosen to correspond to the wider objects (lower part of the spectrum) within the P-QRS-T cycle - the $\mathrm{T}$ waves. Under normal circumstances, $\mathrm{T}$ wave takes about $40 \%$ of the cycle's duration. Based on the actual HR, the SE is dimensioned accordingly. 
Original ECG Signal

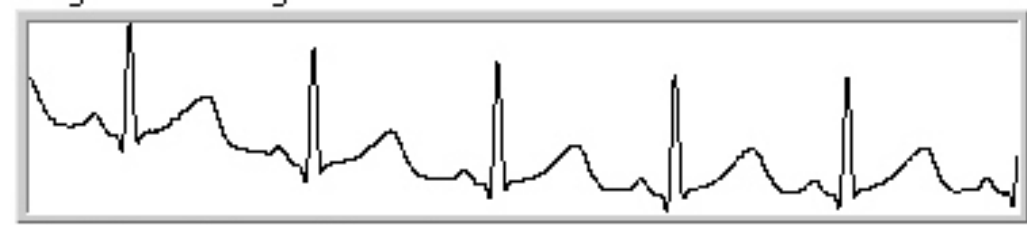

High-Pass Filtered Signal

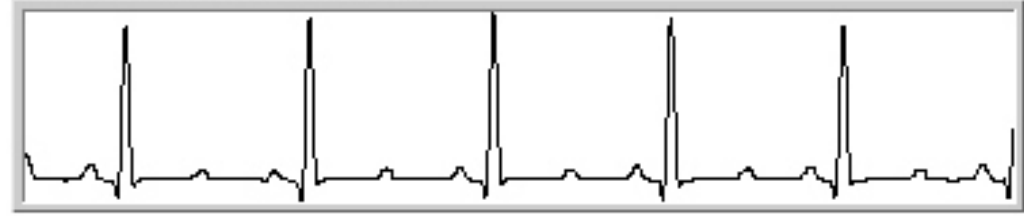

Figure 5: QRS detection using High-Pass MF

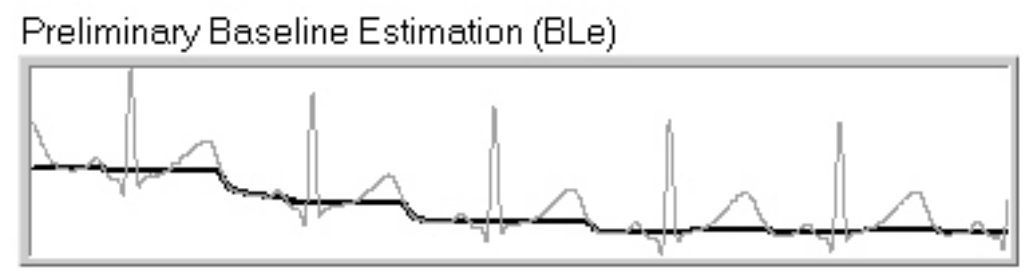

Figure 6: Preliminary baseline estimation

After the preliminary baseline estimation, it is subtracted from the original ECG signal resulting in the PQRST signal. The baseline wander is now sufficiently removed for the process of morphology recognition to begin. Having the R-wave positions and several physiologically based constraints (refractory periods, etc.), the rest of morphology components are determined using several derivation routines. This is illustrated in Figure 7. Final baseline correction (BLc) is made upon correction of P-waves' starting points (Pstart), which is a necessary precaution against all the interference and degradations compromising the acquired ECG signal. Only TP segments can be considered as "truly isoelectric", and only their ending points (Pstart) are secured from ADC settling-time transitions (sometimes visible as non-physiological negative deflections after $\mathrm{T}$ waves, due to the acquisition amplifier filter's inertia). At the end of the correction process, corrected are the Pend and Tend positions.

Figure 8 illustrate $\mathrm{J}$ points being defined after $\mathrm{S}$ deflections in the positions where the first derivative opens a predefined angle (i.e. 60 degrees). 


\section{Morphology Determination}

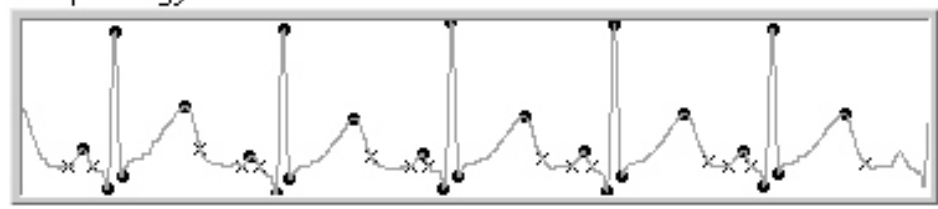

Figure 7: Morphology recognition (Ps,P,Pe,Q,R,S,T,Te)

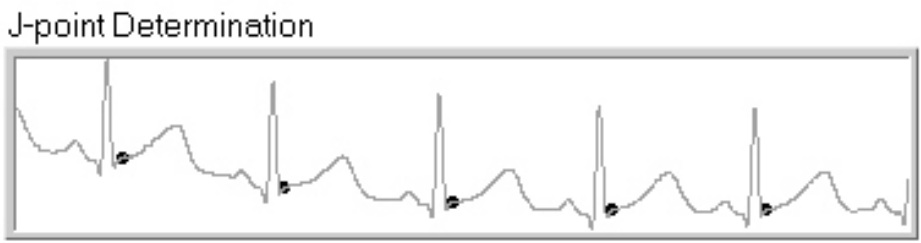

Figure 8: J point determination

A new convention for ST segment elevation is proposed instead of the standard, taking the readings at $80 / 60 \mathrm{~ms}$ after $\mathrm{J}$ point for HR less/higher than $120 \mathrm{bpm}$ respectively. Instead of a fixed interval after $\mathrm{J}$ point which is very inappropriate in cases of extreme arrhythmias, more consistent is to take the readings at time positions relative from distinguished event (i.e. $30 \%$ of RT interval). Also more reliable seems to be an average of a segment at/after the measurement moment than a single sample.

Finally, all relevant amplitudes are read (measured) at the P, R, T and ST positions, and time intervals are calculated for $\mathrm{P}$ width, QRS duration, RR interval, PR interval, QT interval and QTc. For each measurement or calculation, calculated are also its mean value, standard deviation and median value within the analyzed ECG strip, thus applying statistical reliability. Using these statistical values for each measurement within the strip (taken from all P-QRS-T complexes) extreme deviations due to artifacts are automatically rejected. The standard deviation can indicate possible arrhythmia detection.

\section{Discussion}

The proposed morphology filtering shows reliable results for applications where a single lead is tracked. Figure 9 and Figure 10 show prominent examples of MF operation.

This Math. Morphology based ECG analysis system is a perfect solution for on-the-fly CCU monitoring. It is very accurate and reliable and almost 


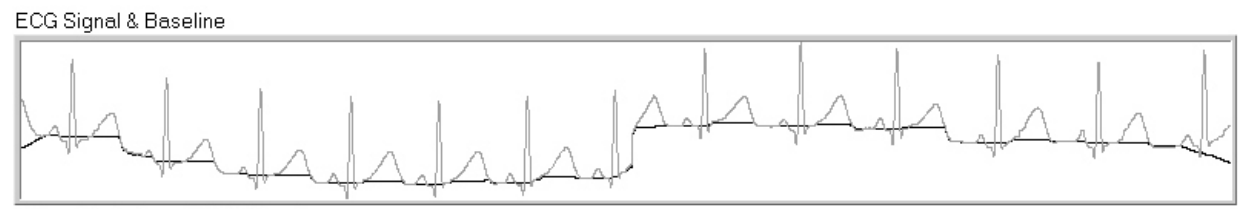

Filtered \& Analyzed ECG Slgnal

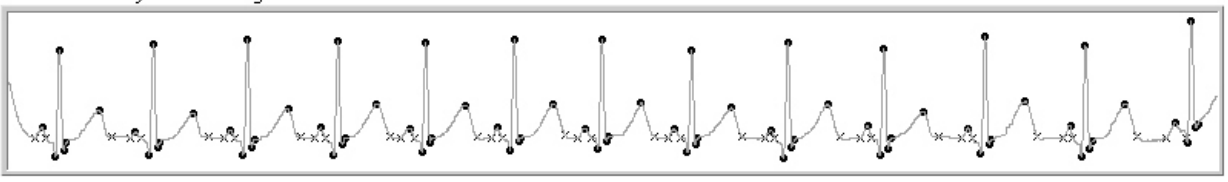

Figure 9: Processed ECG trace

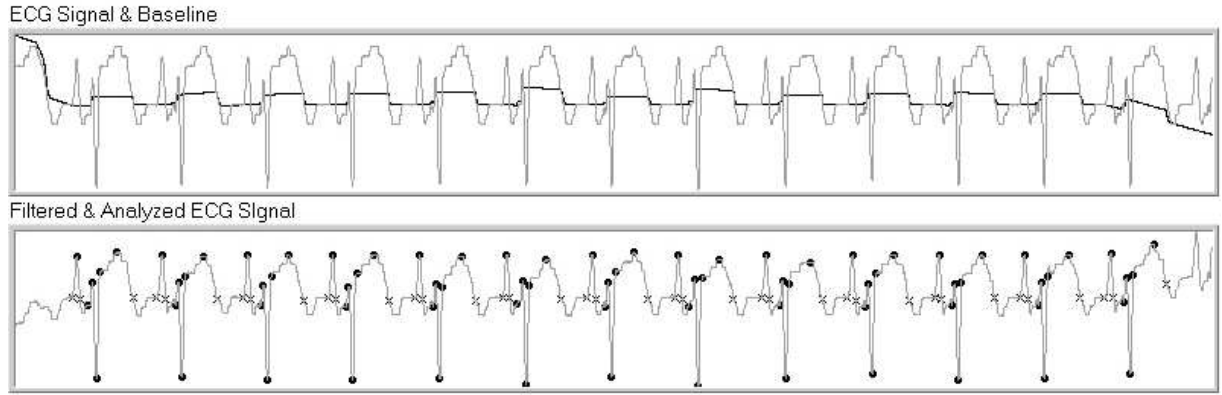

Figure 10: Processed ECG trace

error-proof due to the median filter that protects from artefact driven errors. The semi real-time follow-up of the relevant parameters is achieved by the fast strip analysis. If optimized for speed, on a fast PentiumII or higher, 4-6 processings per minute can be performed which is more than enough for monitoring even in critical cases with infarction development. However, it is obvious that the algorithm is "blind" for any events on the ECG trace that are not related to $\mathrm{R}$ waves. Therefor, no AV blocks can be directly detected. Further more in case of VES (ventricular extra sistolaes) the algorithm will try to reveal the morphology around it. The first case implicates loss of data, however the latter one severe distorted data into the table of parameters. In the first case, less complexes would participate in the table thus the statistical reliability will lower. In the latter case, impossible values will additionally show up in the table and will be ignored by the median filter. So no real damage is implicated. It is obvious though, the standard deviation of the RR interval would show unusually big values indicating a possible arrhythmia in the ECG. Additional algorithm should be developed to distinguish whether Rs are missing (AV block of x:1 type) 
or VES are captured (dangerous if hit into repolarisation period). The latter case should involve the concept of the "refractory periods". This algorithm involves an adaptive approach to the ST elevation estimation. There is no officially adopted procedure for the measured point/region definition, it is always more or less dependent on the operator to decide for, and most likely it is a fixed period after the $\mathrm{J}$ point. A new strategy is proposed within this system, a one based on the actual HR or RR interval. The corresponding module can tie the measurement to the $\mathrm{J}$ point or to the more reliable $\mathrm{S}$ downstroke. The measurement can be performed over the particular point or a segment, and finally the period can be fixed or variable between the $\mathrm{S}$ and $\mathrm{T}$ points.

\section{Conclusion}

The proposed filtering application of Mathematical Morphology for analysis of ECG signals offers a possible solution to most of the problems faced with modern ECG analysis such as morphological identification. The reliable measurements of various elements of the ECG and proper identification of the baseline will offer precise determination of certain time intervals and segments of the cardiac cycle. From this the rapid analysis of short segments will provide a positive degree of statistical confidence in the presented results with the elimination of extreme mistakes due to the exclusion of artifacts. These proposed calculations have the advantage over the currents methods for real-time analysis in that they are not free from the cumulative error from current averaging algorhythms. Keeping in mind today's computation possibilities of computers, the proposed Morphological Filter has a prospect of being a quick and accurate monitor of the ECG signal either as complete cardiac cycle or partial elements.

\section{REFERENCES}

[1] L. Najman, H. Talbot, Mathematical Morphology, From Theory to Applications, ISTE Ltd and John Wiley \& Sons, Inc., 2010.

[2] J. Serra, Image analysis and mathematical morphology, academic press, inc, 1982.

[3] Y. Shih, Image processing and mathematical morphology fundamentals and applications, CRC Press, Taylor \& Francis Group, 2009.

[4] P. Tadejko, W. Rakowski, Mathematical Morphology Based ECG Feature Extraction for the Purpose of Heartbeat Classification, Proceedings Proceedings of the 6th International Conference on Computer Information Systems and Industrial Management Applications, CISIM '07, Pages 322327, June 28 - 30, 2007.

[5] S. Yazdani, J. Vesin, Extraction of QRS fiducial points from the ECG using adaptive mathematical morphology, Digital Signal Processing 56 (2016) 100109. 
110 R. GOLUBOVSKI, GJ. MARKOSKI, E. GOLUBOVSKA, AND V. KOKALANOV

[6] R. Kaur Bal, A. Kumar, Comparative Analysis of Various QRS Techniques in ECG, IJCSMC, Vol. 5, Issue. 2, February 2016, pg.68 75.

[7] Y. Pang, L. Deng, J. Lin, Z. Li, G. Li, Q. Zhou, Removal Method of Baseline Drift from ECG Signals Based on Morphology Filter, Applied Mechanics and Materials, Vols 427-429 (2013) pp 1691-1695.

Faculty of Natural Sciences and Mathematics, Ss. Cyril and Methodius University, Skopje, Macedonia

E-mail address: roman.golubovski@t.mk

Institute of Mathematics, Faculty of Natural Sciences and Mathematics, Ss. Cyril and Methodius University, Skopje, Macedonia

E-mail address: gorgim@pmf.ukim.mk

E-mail address: elena.golubovska@gmail.com

Faculty of Computer Science - University Goce Delchev, Shtip, MacedoNIA.

E-mail address: vasko.kokalanov@ugd.edu.mk 